\section{Wird das Risiko für eine Leukämie durch Impfungen erhöht?}

Im Jahr 2002 sorgte ein auf einem amerikanischen Krebskongress gezeigtes Poster für großes Aufsehen. Darin wurde die Hypothese aufgestellt, die Hepatitis-B-Impfung erhöhe das Risiko für die Entstehung einer akuten Leukämie. Deswegen wurde in Frankreich eine Fall-Kontroll-Studie durchgeführt, die herausfinden sollte, ob Impfungen wirklich das Risiko für akute Leukämien erhöhen.

D ie akute Leukämie ist weltweit die häufigste Krebserkrankung im Kindesalter, wobei die Inzidenz bei den 2- bis 5-jährigen Kindern am höchsten ist. Unter den wenigen bisher identifizierten Risikofaktoren für eine Leukämie finden sich hohe Dosen einer ionisierenden Bestrahlung, einige chemotherapeutische Behandlungsstrategien und bestimmte vererbbare Syndrome wie der Morbus Down. Laufende Forschungsprojekte untersuchen weitere potenzielle Risikofaktoren, wie z. B. Infektionen und Impfungen.

Die nationale populationsbasierte FallKontroll-Studie aus Frankreich wurde zwischen 2003 und 2004 durchgeführt. In die Analyse wurden insgesamt 776 Patienten mit akuter Leukämie und 1.681 Kontrollen aufgenommen. Die Daten hinsichtlich der Impfung (Art der
Impfung, Anzahl der verabreichten Dosen etc.) wurden in einem standardisierten Telefoninterview mit den Eltern erhoben, das identisch für Patienten und Kontrollen war.

Insgesamt fand sich keine Assoziation zwischen einer Impfung und dem Risiko einer akuten Leukämie, auch nicht bei der Analyse mit den Untergruppen akute lymphatische und akute myeloische Leukämie. Dies galt auch für jede einzelne Impfung selbst, egal ob Totimpfung wie gegen Diphtherie, Tetanus, Poliomyelitis, Pertussis, Hepatitis, Haemophilus influenzae B, Pneumokokken und Meningokokken oder Lebendimpfung wie gegen Mumps, Masern oder Röteln.

Zudem wurde keinerlei Einfluss der Anzahl der jeweils verabreichten Impfdosen, der Anzahl der insgesamt verabreichten Injektionen oder der Anzahl der ins- gesamt verabreichten Impfungen gesehen. Zusätzliche, die Ergebnisse beeinflussende Variablen („Confounder") wurden während der Analyse keine gefunden.

Die Autoren folgern, dass es entsprechend den Studienergebnissen keine Evidenz gibt, dass Impfungen das Risiko für eine akute Leukämie erhöhen.

Kommentar: Die Ergebnisse dieser Arbeit sind wichtig für die Entkräftung des Argumentes der Impfgegner, Impfungen würden das Risiko einer akuten Leukämie erhöhen. Impfungen stehen mit akuten Leukämien nur im zeitlichen, jedoch nicht im kausalen Zusammenhang. Obwohl eine statistische Aufarbeitung dieser Fragestellung insgesamt schwierig ist und viele abhängige und unabhängige Variablen mitbewertet werden müssen, konnten die Ergebnisse dieser Arbeit in einer zeitnah erschienenen Veröffentlichung einer kanadischen Arbeitsgruppe von Amy MacArthur bestätigt werden [Am J Epidemiol 2008; 167: 598-606].

Prof. Dr. Thomas Lehrnbecher

Mallol-Mesnard $\mathrm{N}$ et al. Vaccination and the risk of childhood acute leukaemia: the ESCALE study (SFCE). Int J Epidemiol 2007; 36: 110-6

\section{Erdnüsse an Schwangere - Allergieprophylaxe für die Kinder?}

Lange Zeit wurde gelegentlich „aus dem Bauch heraus“ empfohlen, zur Allergieprophylaxe auf Erdnüsse in Schwangerschaft und Stillzeit zu verzichten. Seit 2009 raten nun offizielle Leitlinien von jeglicher Restriktion bei der Nahrung mit dem Ziel der Allergieprävention ab.

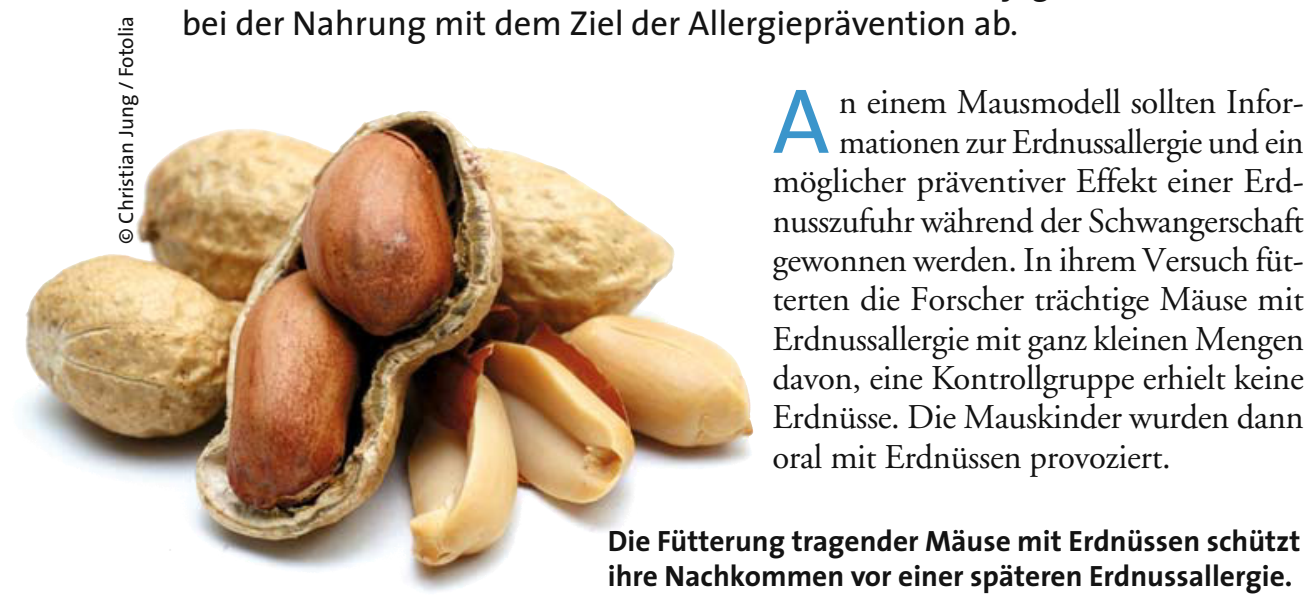

Während die Nachkommen der Mäusefamilien mit pränataler Erdnussexposition in 28\% der Fälle allergische Reaktionen zeigten, hatte die Kontrollgruppe eine Quote von 80\%. Die erste Gruppe der Mäusebabys zeigte dabei auch erhöhte Spiegel an spezifischem, schützendem $\operatorname{IgG}_{2 \mathrm{a}}$.

Kommentar: Kleine Mengen von Erdnuss während der Schwangerschaft können also das Risiko einer Anaphylaxie mindern. Ob sich diese Ergebnisse bei Mäusen auf den Menschen übertragen lassen, ist bislang offen.

Dr. Ulrich Mutschler

López-Expósito l et al. Maternal peanut exposure during pregnancy and lactation reduces peanut allergy risk in offspring. J Allergy Clin Immunol 2009; 124: 1039-46 